\title{
Geometry of Non-expanding Horizons and Their Neighborhoods
}

\author{
Jerzy Lewandowski
}

December 9, 2018

Instytut Fizyki Teoretycznej, Uniwersytet Warszawski, ul. Hoza 69, 00-629 Warszawa, Poland

\begin{abstract}
This is a contribution to MG9 session BHT4. Certain geometrically distinguished frame on a non-expanding horizon and in its space-time neighborhood, as well as the Bondi-like coordinates are constructed. The construction provides free degrees of freedom, invariants, and the existence conditions for a Killing vector field. The reported results come from the joint works with Ashtekar and Beetle [2].
\end{abstract}

In the quasi-local theory of black holes proposed recently by Ashtekar [1] a $\mathrm{BH}$ in equilibrium is described by a 3 -dimensional null cylinder $\mathcal{H}$ generated in space-time by null geodesic curves intersecting orthogonally a spacelike, 2-dimensional closed surface $S$. The standard stationarity of space-time requirement is replaced by the assumption that the cylinder has zero expansion, that is $\mathcal{H}$ is a non-expanding horizon. This implies, upon the week and the dominant energy conditions, that the induced on $\mathcal{H}$ (degenerate) metric tensor $q$ is Lie dragged by a null, geodesic flow tangent to $\mathcal{H}$. The geometry induced on $\mathcal{H}$ consists of the metric tensor $q$ and the induced covariant derivative $\mathcal{D}$. It is enough for the mechanics of $\mathcal{H}[1]$. The geometry of a non-expanding horizon is characterized by local degrees of freedom. They are an arbitrary 2-geometry of the null generators space $S$, the rotation scalar, and certain tangential 'radiation' evolving along the horizon. 
In the standard, Kerr-Newman case, the event horizon is equipped with a null Killing vector field. In our general non-expanding horizon case, however, a Killing vector field may not exits at all. Our first goal is a geometric condition which distinguishes a null vector field $\ell_{0}$ tangent to $\mathcal{H}$ and which is satisfied by the Killing vector field whenever it exists. We a made extra assumptions about the stress energy tensor at $\mathcal{H}$ that are satisfied for the Maxwell and/or scalar and/or dylaton fields. The condition distinguishing the null vector field $\ell_{0}$ was obtained by making as many components of the tensor $[\ell, \mathcal{D}]_{b c}^{a}$ defined on $\mathcal{H}$ as possible zero, as we vary $\ell$. But here we give a more geometric definition of this choice. Due to the evolution equations of $\mathcal{D}$ along $\mathcal{H}$, there is a unique extension $(\tilde{\mathcal{H}}, \tilde{q}, \tilde{\mathcal{D}})$ of $(\mathcal{H}, q, \mathcal{D})$ in an affine parameter along the null geodesics. We claim, that generically $\tilde{\mathcal{H}}$ admits a unique global crossection $S_{0}$ such that its expansion in the transversal null direction orthogonal to $S_{0}$ (this information is contained in $\tilde{D}$ ) is zero everywhere on $S_{0}$. Given the crossection $S_{0}$, there is a unique null vector field $\ell_{0}$ vanishing identically on $S_{0}$ and such that $\mathcal{D}_{\ell_{0}} \ell_{0}=\kappa_{0} \ell_{0}, \kappa_{0} \neq 0$ being a constant. Fixing some value $\kappa_{0}(q, \mathcal{D})$ determines $\ell_{0}$ completely. The shear of $S_{0}$ vanishes in the null transversal direction orthogonal to $\tilde{\mathcal{H}}$, iff $\ell_{0}$ generates a symmetry of the geometry $(q, \mathcal{D})$. The commutator $\left[\mathcal{L}_{l_{0}}, \mathcal{D}\right]$ represents the tangential radiation, and $\mathcal{H}$ is not a Killing horizon unless the comutator is zero.

The rotation 1-form potential $\omega_{0}$ of $\ell_{0}$ is defined by $\mathcal{D} \ell_{0}=\omega_{0} \otimes \ell_{0}$. We define a good cut as a space-like section of $\mathcal{H}$ such that the pullback of $\omega_{0}$ thereon is a harmonic 1 -form. The good cuts define a foliation of $\mathcal{H}$ invariant with respect to the flow of $\ell_{0}$, owing to $\mathcal{L}_{\ell_{0}} \omega_{0}=2 d \kappa_{0}=0$.

Given $\ell_{0}$ and the good cuts foliation, we determine a null frame $\left(m_{0}, \bar{m}_{0}, n_{0}, \ell_{0}\right)$ by using another null vector $n_{0}$ orthogonmal to the lives requiring $n_{0 \mu} \ell_{0}{ }^{\mu}=$ -1 , and $\operatorname{Re}_{0}{ }^{\mu} K_{, \mu}=0$ where $K$ is the Gauss curvature of $\mathcal{H}$, generically non-constant.

In a neighborhood of $\mathcal{H}$, the good cuts foliation and the distinguished $\ell_{0}$ define a unique geodesic extension of the vector field $n_{0}$. It is used to extend the foliation and frame to the neighborhood.

The applications and results of this construction are $a$ ) invariants of the horizon and of the neighborhood, $b$ ) invariant characterization and true degrees of freedom of a horizon and of its neighborhood in the vacuum or Maxwell and/or scalar and/or dylaton case, $c$ ) classification of the symmetric isolated horizons, $d$ ) necessary and sufficient conditions for the existence 
of a Killing vector field, and the control on the space-times not admitting a Killing vector field.

\section{Acknowledgments}

This research was supported in part by Albert Einstein MPI, CGPG of Pennstate University, and the Polish Committee for Scientific Research under grant no. 2 P03B 06017.

\section{References}

[1] Ashtekar A., Beetle C., Dreyer O., Fairhurst S., Krishnan B., Lewandowski J., Wiśniewski J., Phys.Rev Let.853564(2000), grqc/0006006, See the contribution by Olaf Dreyer in the same session

[2] Ashtekar A., Beetle C., Lewandowski J. Space-Time Geometry of Isolated Horizons I,II, in preparation 